
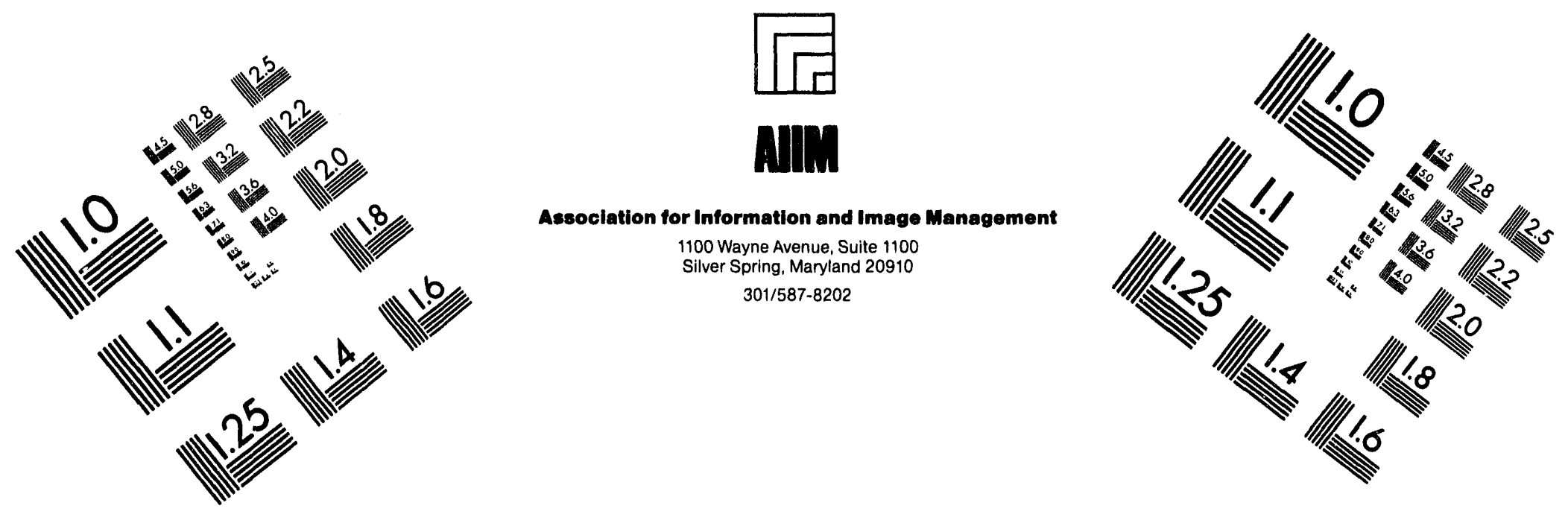

\title{
Centimeter
}

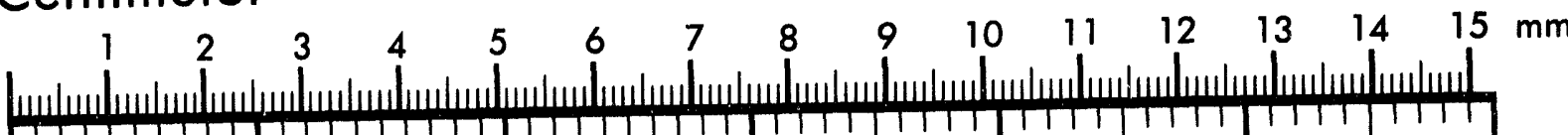

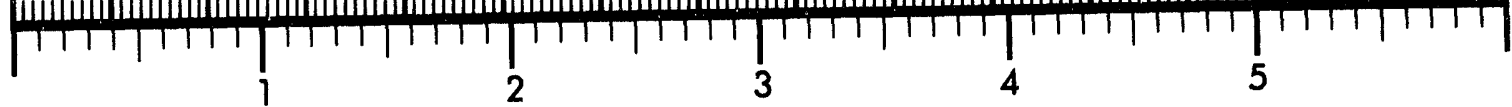
Inches
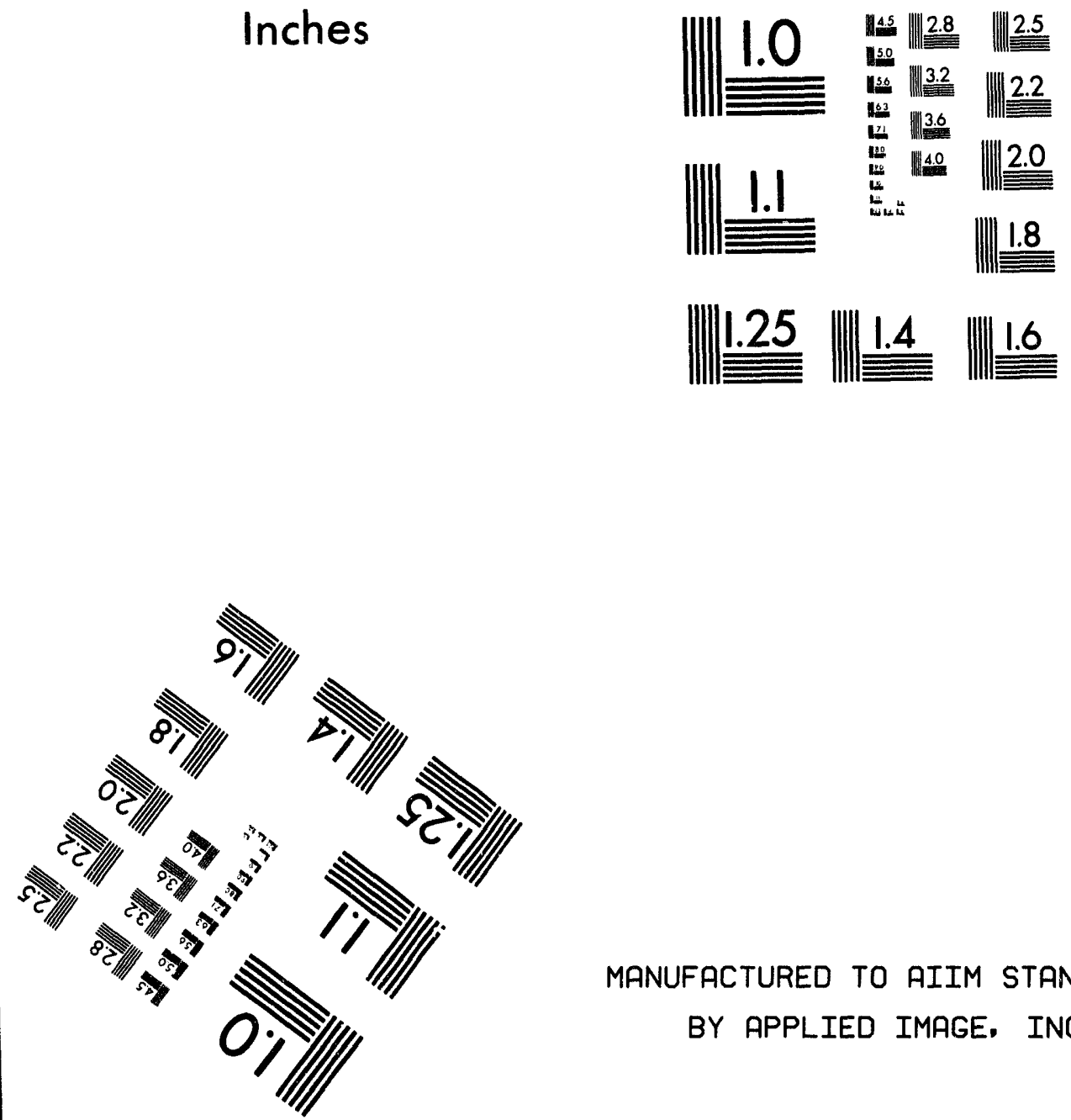

MANUFACTURED TO AIIM STANDARDS

BY APPLIED IMAGE, INC.

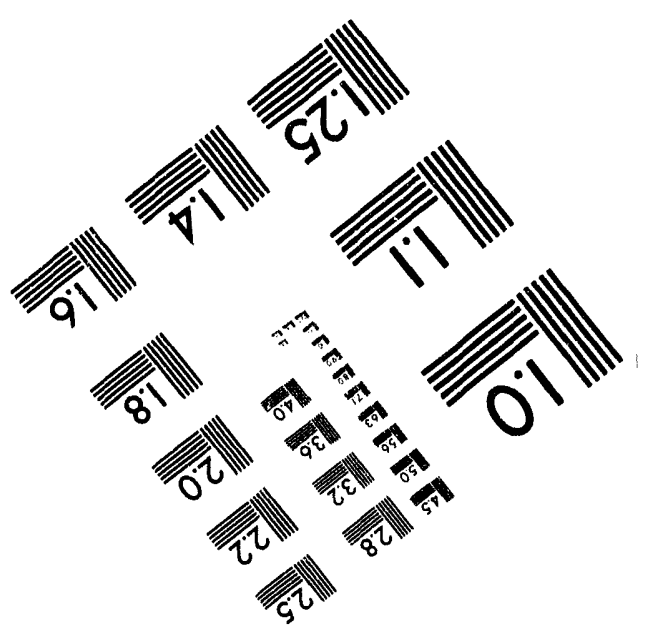


:
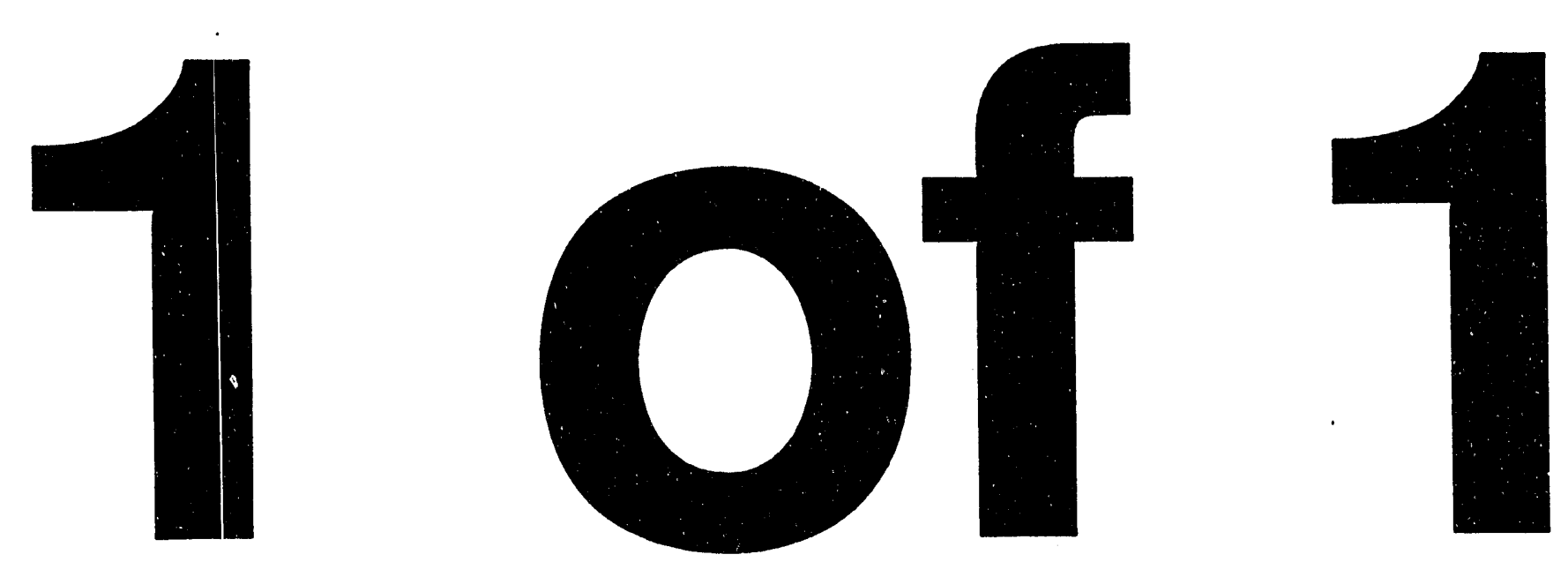


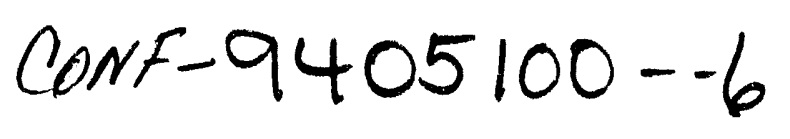

The ubmitted manucript hos been authored by - contrector of the U.S. Governmem under contrect No. DEACO5-840A2 1400. Accordingty, the U.S. Governmem roteine a nonexectuaive. rovatififree licemese to publeth of reproduce the publishad form of this contribution. or thew others to do so. for U.S. Government purposes."

\section{First Principles Simulation of Materials Properties}

\author{
W. A. Shelton, G. M. Stocks \\ Oak Ridge National Laboratory \\ Oak Ridge, TN 37831-6367
}

\author{
R. G. Jordan, Y. Liu, L. Qui \\ Florida Atlantic University \\ Boca Raton, FL 33431-0991
}

\author{
J. B. Staunton \\ University of Warwick \\ Coventry CV4 7AL, UK
}

\author{
D. D. Johnson \\ Sandia National Laboratory \\ Livermore, CA 94551-0969 \\ B. Ginatempo \\ Uninversita' di Messina \\ I-98166 Agata-Messina, Italy
}

\begin{abstract}
We have developed a hybrid, parallel computer code for calculating the electronic structure of both ordered and substitutionally disordered materials. By using PVM3.3, we can integrate into our local computer environment multiple parallel and vector supercomputers as well as high performance workstations. Without this approach, calculations of materials properties of large systems would be otherwise untenable due to a lack of computer resources. For example, we have determined the short-range order intensity and its electronic origin for the $\mathrm{Ag}-\mathrm{Mg}$ alloy system, including an estimate of the order-disorder (spinodal) temperature.
\end{abstract}

\section{Introduction}

The design of new materials with highly desirable physical properties rests largely on a set of rules and parameters set forth originally by Hume-Rothery. These rules have provided metalurgists with a basis for understanding phase stability in alloys. This approach, though successful, is a time consuming and economically expensive process that in the end may yield a material lacking the desired physical properties.

The development of local density approximation (LDA) based first-principles, electronic-structure methods for studying the physical properties of real materials has emerged as an important component in the design of new materials. The advantage of this approach is that the properties, energetics, and mechanisms responsible for phase transformations do not depend on input parameters. Because these methods are parameter free, they are predicative methods that can be used to aid the metallurgist and alloy designer in the development of new materials.

A large amount of computer resources is necessary to calculate the physical properties of both ordered and substitutionally disordered materials using a firstprinciples approach. The amount of cpu time for one iteration of a small system containing five atoms per unit cell running at $90 \%$ peak performance $(2.35 \mathrm{gi}$ gaflops) on a Cray YMP/8 is one hour. Running the same size problem on a 128-node Intel iPSC/860 hypercube is slightly better at 2.5 gigaflops, but the limiting factor on the iPSC/860 is the 8 Mbytes of memory per node. Given that the problem size scales as $N^{3}$ (where $N$ is the number of atoms in the unit cell), the amount of cpu time and memory required for calculations on medium size systems containing tens of atoms per unit cell would dramatically increase rendering the problem intractable.

Parallel Virtual Machine (PVM) is a messagepassing system allowing for different machine architectures on various networks to be linked together into a heterogenous, distributed computing environment which to the user is seen as a single computational tool. An attractive feature of PVM is its ease of use. For those already familiar with message passing, it is a lesson in syntax; while for the beginner PVM provides illustrative examples in Fortran and C enabling a quick startup. By using PVM3.3 we are able to link together various types of supercomputers and high performance workstations for the possibility of solving large scale problems (10's to 100 's of atoms per unit cell) in material science as well as other computationally challenging problems. In addition, we are able to avoid problems with loading a large code on platforms with a small amount of local memory. This is done by rewriting the code using a master/slave programming paradigm where the master resides on a particular node in the computing environment that

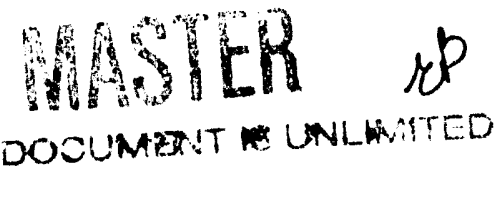


has an ample amount of memory.

During recent years, significant progress towards the development of first-principles, electronicstructure methods that describe the properties of substitutionally disordered alloys has been realized $[8,9$, 10]. More recently, calculations for multisublattice substitutional alloys were performed using the LDAbased, multiatom per unit cell, scalar-relativistic, selfconsistent-field, Korringa, Kohn and Rostoker, coherent potential approximation (LDA-SCF-KKR-CPA) method [25][5][22]. The LDA is used to treat the many-body quantum mechanical effects of electron exchange and correlation. The CPA is used to handle the effects of random substitutional disorder on the electronic structure within the multiple-scattering formalism of the Green's-function-based KKR method. The relativistic effects are treated within the socalled scalar-relativistic approximation, which omits the spin-orbit term but includes the mass-velocity and Darwin terms.

By studying the energetics of nearby disordered phases it is possible to uncover the electronic mechanisms that are responsible for the formation of specific ordered phases. This knowledge would provide insights into possible ways of obtaining desirable phases or suppressing undesirable ones. The approach used in this paper is to search for an instability of the disordered phase to some type of ordering, i.e. short-range order, which can be a precursor to the low temperature behavior. As an example of this approach, we discuss our calculations of the short-range order, its origin, and the transition temperature of $\mathrm{Ag}_{75} \mathrm{Mg}_{25}$.

In the following sections, a brief description of the theoretical foundation of the LDA-SCF-KKR-CPA method, the parallelization, theory of compositional ordering and results will be given.

\section{LDA-SCF-KKR-CPA}

\subsection{Local Density Approximation}

The density functional theory (DFT) is an exact method for calculating the energetics of the manybody quantum mechanical system consisting of the electrons in the field of the atomic nuclei [7]. The foundation of this theory is that the total energy of the system is a unique functional of the electron charge density and at its minimum yields the true ground state total energy and electron charge density. At the heart of this formalism is the calculation of a non-local, exchange-correlation potential that accounts for the many-body effects of electron exchange (Pauli-exclusion principle or exchange energy) and electron correlation. However, the non-local exchange-correlation potential is unknown, requiring further approximation.

The local-density approximation to density functional theory is a technique for treating the nonlocal exchange-correlation potential within a meanfield theoretical framework, resulting in a set of singlebody Schrodinger-like equations (Kohn-Sham equations) with an effective potential containing a local exchange-correlation potential [17]:

$$
\left[-\nabla^{2}+V^{e f f}(\vec{r})\right] \psi(\vec{r}, \epsilon)=\epsilon \psi(\vec{r}, \epsilon) .
$$

It should be noted that the DFT-LDA theory is a ground-state theory. The eigenvalues for the KohnSham equations only represent the true eigenvalues of the system in its ground state and are not the true eigenvalues for the excited states.

\subsection{Korringa, Kohn and Rostoker Method}

The Korringa, Kohn and Rostoker (KKR) method is a Green's function based theory for calculating the electronic structure of periodic crystalline materials. There are two approaches used to obtain the KKR equations. The first approach developed by Korringa is based on multiple scattering theory [18], while the second method was developed by Kohn and Rostoker and uses a variational technique [16]. We adopt the multiple-scattering approach of Korringa.

The KKR method makes use of scattering theory to calculate the scattering path operator which is used to construct the single-site Green's function:

$$
\begin{array}{r}
G_{i s}^{\alpha}(\vec{r}, \vec{r}, \epsilon)=\sum_{L, L^{\prime}}\left[Z_{L}^{\alpha}(\vec{r}, \epsilon) \tau_{L L^{\prime}}^{\alpha, i s, i s}(\epsilon) Z_{L^{\prime}}^{\alpha}(\vec{r}, \epsilon)\right. \\
\left.-Z_{L}^{\alpha}(\vec{r}, \epsilon) J_{L^{\prime}}^{\alpha}(\vec{r}, \epsilon) \delta_{L L^{\prime}}\right],
\end{array}
$$

$Z_{L}^{\alpha}(\vec{r}, \epsilon) \equiv$ Regular single-site wave function of the $\alpha^{\text {th }}$ atomic specie.

$J_{L}^{\alpha}(\vec{r}, \epsilon) \equiv$ Irregular single-site wave function of the $\alpha^{t h}$ atomic specie.

$\tau_{L L}^{\alpha, i s, i s}(\epsilon) \equiv$ Single-impurity scattering-path operator of the $\alpha^{\text {th }}$ atomic specie.

The scattering-path operator describes the conversion of an incoming electron wave function at the $i^{\text {th }}$ site into an outgoing one at the $j^{\text {th }}$ site and includes all possible scattering paths (multiple scattering). A schematic representation of this physical process is shown in figure 1. It is assumed that a crystalline solid is composed of an infinite number of atoms. Because 


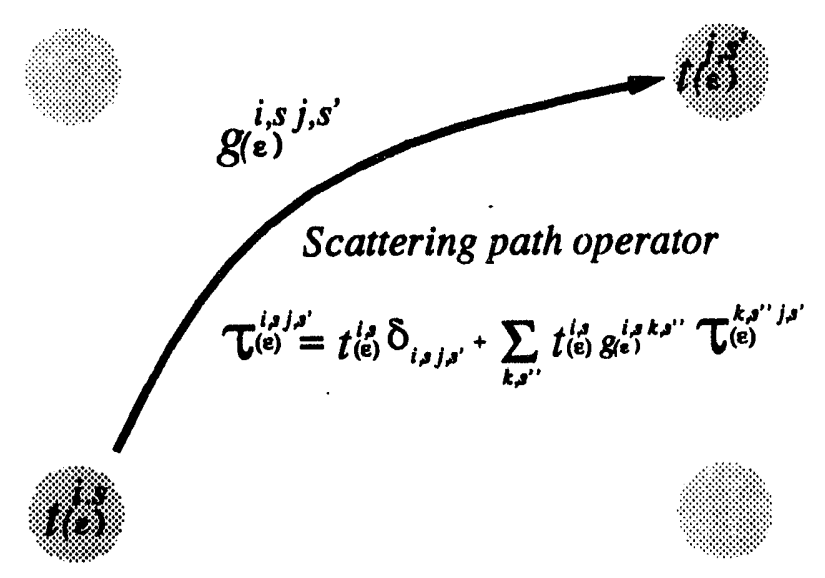

Describes the conversion of an incoming electron wave function at site $i$ into an outgoing one at site $j$ and includes all possible scattering paths between the two sites.

Figure 1: Schematic representation of the scattering process

the system is periodic a lattice Fourier transform is performed resulting in

$$
\left.\tau_{c, L L^{\prime}}^{0,0, \boldsymbol{s}}=\frac{1}{\Omega_{k}} \int_{\Omega_{k}} d^{3} k\left[t_{c, \ell}^{s, 0}(E)^{-1}\right\}-G_{L L^{\prime}}^{s,,^{\prime}}(\vec{k}, E)\right]^{-1},
$$

requiring an explicit matrix inversion at each $\vec{k}$ point on a 3-dimensional mesh; this is the time consuming step of the calculation.

There are three major advantages in choosing this method over other band theoretical techniques. Firstly, the best method for calculating the effects of random substitutional disorder on the electronic structure is the coherent potential approximation. This method requires the Green's function which the KKR yields naturally and directly. Secondly, there exists a natural separation between the underlying lattice structure and potential. This can be seen from the above equation. $t_{c, \ell}^{s, 0}(E)^{-1}$ is independent of the spatial coordinates and depends only on the potential through the scattering phase shifts. The phase shifts are obtained by solving the Schrodinger equation (or Dirac equation for relativistic systems). The information about the lattice structure is contained in $G_{L^{\prime} L^{\prime}}^{s^{\prime}}(\vec{k}, E)$ which is a free electron Green's function and describes how a free-electron is propagated from the $i^{\text {th }}$ site to the $j^{\text {th }}$ site. The significance of this separation is that the disorder is site-diagonal. In other band theory methods there exist off-diagonal disorder that is difficult to treat in the CPA requiring further approximation. Thirdly, to determine possible mechanisms responsible for certain properties of an alloy and to calculate its transition temperature necessitates the calculation of the susceptibility and to determine it requires the Green's function of the system.

\subsection{Coherent Potential Approximation}

The coherent potential approximation is used to calculate the effects of random substitutional disorder on the electronic structure. Substitutional disorder refers to the random occupation of the atomic sites by the alloying components on a underlying fixed crystal structure. The CPA replaces the randomly arranged potentials by an effective one, and thereby restores the periodicity that is necessary to perform electronic structure calculations.

The CPA is an analytic mean-field theory for calculating the configurationally averaged Green's function from which the physical properties of the disordered alloy can be easily calculated. It is exact in the weak and strong scattering limits and is exact in the limit the concentration approaches zero or one. The CPA is considered the best single-site method for treating the problem of random substitutional disorder [26] [24][25].

The CPA equations are written as,

$$
\sum_{\alpha} c_{\alpha}^{s} \tau_{\alpha, L L^{\prime}}^{0,0, s}(E)=\tau_{c, L L^{\prime}}^{0,0, s}(E)
$$

The condition on the CPA is that the coherent scattering-path operator equals the average of the single-site impurity path operators. A single-impurity scattering path operator is obtained by replacing one of the coherent potentials by one of the atomic potentials. This process is repeated for each atomic constituent yielding $n$ single impurity scattering path operators. Each single-impurity path operator can be interpreted as a perturbation on the CPA medium with the CPA equations requiring that the change in scattering produced by these perturbations vanish upon averaging (see figure 2).

The CPA equations are solved by an iterative method that has been shown [20][23] to always converge provided that the initial guess to $t_{c, l}^{d, 0}(E)$ is the average t-matrix approximation (ATA):

$$
t_{c, \ell}^{s, 0}(E)=t_{\ell}^{A T A, s}=\sum_{\alpha} c_{\alpha}^{s} t_{\ell}^{\alpha, s} .
$$

A flow chart of the CPA iterative scheme is given in figure 2 . 


\section{CPA}

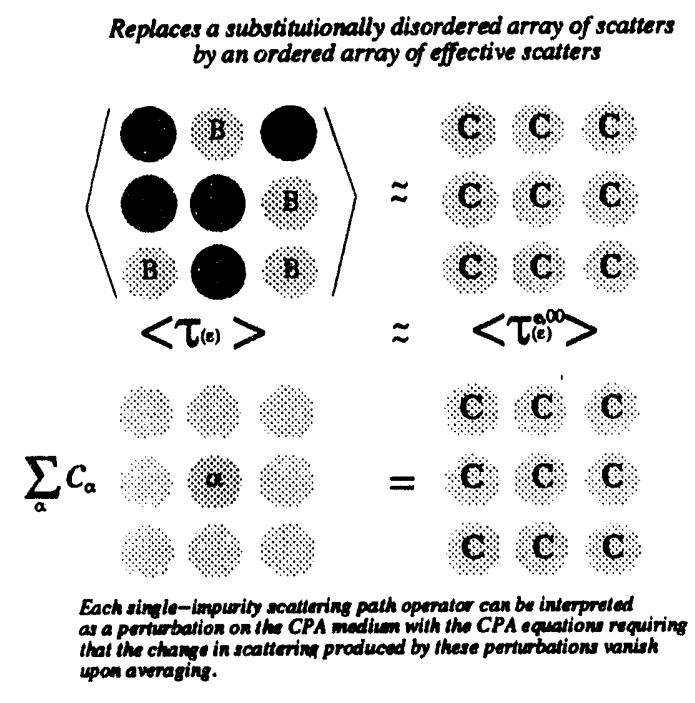

Figure 2: Schematic representation of the CPA

INPUT A'I'A for guess to $t_{c}$

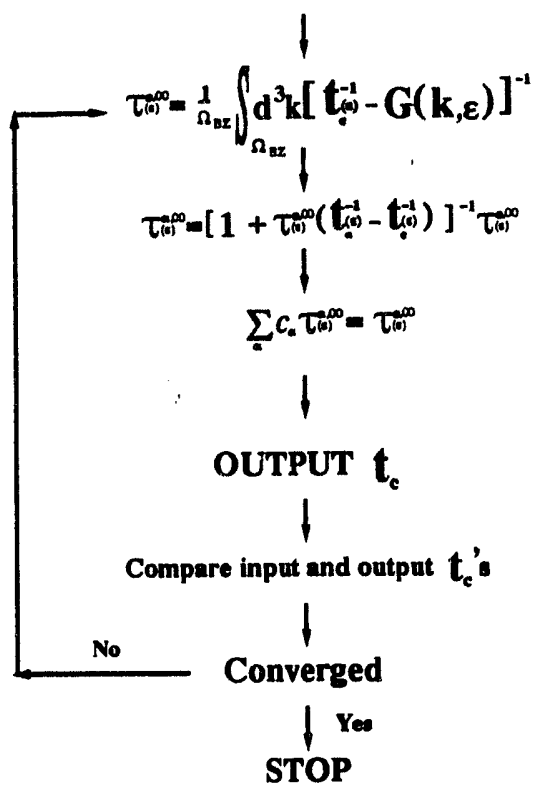

Figure 3: Flow chart of the CPA iterative procedure

\section{Parallelization}

At each step of the self-consistency loop the accurate determination of the Fermi energy, charge density, potential and total energy is necessary. To calculate these quantities requires the integration of the singlesite Green's function over the energy contour. The calculation of this function at each point on the contour can be performed independently, highlighting a coarse grain, highly efficient parallel algorithm. In addition, at each energy grid point the scattering path operator must be calculated. Part of this calculation requires an explicit inverse at each of the $\vec{k}$-points. A second strategy of parallelization can be realized by noting that the explicit inverse at each $\vec{k}$-point (or group of $\vec{k}$-points) can be done independently. However, for this paper we adopt the first strategy for implementing the parallelization.

A master/slave paradigm is used where the master has all the information about the problem to be solved (eg. alloying components, crystal structure, magnetic state, etc...) while the slaves only communicate and receive work tasks from the master. The master program reads in a simple input file containing the information necessary to set up the problem. The information in the input file, which includes the input potential and crystal structure, is passed to the slaves. The master program sets up the energy contour and each slave receives an energy grid point. The slaves work task is to calculate the single-site Green's function and density of states for all the alloying constituents. This information is passed back to the master and is used to calculate the Fermi energy, charge density and total energy. From the charge density a new potential is constructed for the next self-consistent iteration. This process is repeated until the difference between sucessive iterations of the total energy is less than 0.1 mRy. At this stage the calculation has reached the self-consistent solution and the program terminates.

\section{Compositional Ordering}

In this section, a theory for compositional ordering will be presented. This theory is quite general; it is capable of describing both commensurate- and incommensurate-type ordering waves, or modulations [15]. These modulations typcially give rise to the phase transformation from the compositionally disorder phase to the order phase. A commensurate modulation has an associated wave-vector whose wave length equals a real-space translation vector. In addition, the theory not only describes Fermi-surface 
mechanisms but also mechanisms that do not depend on the details of the Fermi surface but still rely on the electronic structure $[6,27,11]$. Most importantly, the theory is used to calculate physical quantities that can be measured experimentally, providing a direct correspondence between theory and experiment.

The diffuse scattering intensity $I(\vec{k})$ of electrons from a random binary alloy is proportional to the correlation function $q(\vec{k})$ :

$$
I(\vec{k})=\left(f_{A}-f_{B}\right)^{2} q(\vec{k})=\left(f_{A}-f_{B}\right)^{2} c(1-c) \alpha(\vec{k})
$$

where $f_{A}\left(f_{B}\right)$ are the scattering amplitudes of the $A$ (B) atoms making up the alloy and $\alpha(\vec{k})$ is the WarrenCowley short-range order parameter [3]. The WarrenCowley parameter is related to the lattice Fourier transform of the pair correlation function:

$$
\alpha(\vec{k})=\frac{N}{c(1-c)} \sum_{n}\left\langle\sigma_{0} \sigma_{n}\right\rangle \exp \left(i \vec{k} \vec{R}_{n}\right)
$$

where $\mathbf{N}$ is the total number of atoms. The pair correlation function $\left\langle\sigma_{0} \sigma_{n}\right\rangle$ describes the deviation from the average probability (which for a random substitutional alloy is the concentration of the atomic components) for site pair occupancies. For example, if the alloy is truly random, then for the $i^{\text {th }}$ component the probability of occupancy of each site is the same and is uncorrelated with the remaining sites. The first term $\left\langle\sigma_{0} \sigma_{0}\right\rangle$ is non-zero and equals $c(1-c)$ which produces a smooth background in the diffuse scattering that decreases with scattering angle. On the other hand, any correlation of occupancy between neighboring sites will modulate the background producing satellite peaks [1]. Lifshitz, using a phenomenological approach based on Landau theory [19], has shown that instabilities arise in the homogeneously disordered system at specific $\vec{k}$ vectors which are responsible for driving the phase transition to the ordered state. In additon, these $\vec{k}$ vectors are determined by the space group of the substitutionally disordered alloy[19] requiring no knowledge of the forces involved. These $\vec{k}$ vectors give rise to the satellite peaks observed in diffusive electron scattering experiments. However, this analysis is restricted to systems where these wave vectors are commensurate with the underlying lattice. To investigate systems where incommersurate behavior is responsible for driving the instabilities requires detailed knowledge of the microscopic interactions. The approach that will be described is quite general and can be applied to incommersurate and commersurate behavior. Györffy and Stocks have developed a microscopic theory of compositional ordering that is based on multiple scattering [6][4]. This theory is a meanfield description of the grand potential in the homogeneously disordered system. In this system each alloying species probability of occupancy at a site is equal to its concentration and is the same for all sites. The basis of the theory is the calculation of the fluctuations about the mean-field grand potential due to variations in the site concentration. The variation of the grand potential with respect to small changes in the site concentration describes the symmetry breaking of the translationally invariant system with regard to concentration by the fluctuation in site concentration. This approach has been extended by Staunton, Johnson, and Pinski $[27,11]$ to include terms beyond just the band-energy, i.e. electrostatics and exchangecorrelation terms, as well as improvements to conserve diffuse intensity over the reduced zone in reciprocalspace. This latter improvement is important for setting the temperature scale of the interactions, especially for long-ranged interactions like Fermi-surface effects [27,11]. A central result from this final theory is the direct relationship between $\alpha(\vec{k})$ and the direct correlation function $S^{2}(\vec{Q})$ :

$$
\alpha(\vec{Q})=\frac{1}{1-\beta c(1-c)\left[S^{2}(\vec{Q})-\Lambda\right]},
$$

where $\beta=\left(k_{B} T\right)^{-1}\left(k_{B}\right.$ is the Boltzman constant $)$ and $\Lambda$ is a temperature-dependent, $Q$-independent number calculated within the formalism to conserve spectral intensity of $\alpha(\vec{Q})$, as detailed in $[27,11]$. As can be seen from the above equation, any structure in $S^{2}(\vec{Q})$ will also appear in $I(\vec{Q})$, providing a direct link between theory and experiment. In addition, under certain simplifying approximations (see [4]), $S^{2}(\vec{Q})$ can be written as,

$$
\approx \sum_{\vec{k}} \int d \epsilon \int d \epsilon^{\prime} \frac{f(\epsilon)-f\left(\epsilon^{\prime}\right)}{\epsilon-\epsilon^{\prime}} A^{B}(\vec{k}, \epsilon) A^{B}\left(\vec{k}+\vec{Q}, \epsilon^{\prime}\right)
$$

where $A^{B}(\vec{k}, \epsilon)$ is the Bloch spectral function that represents the density of electron states at energy $\epsilon$ and wave vector $\vec{k}$. In an ordered structure $A^{B}(\vec{k}, \epsilon)$ is a sum of Dirac delta functions at the Bloch eigenvalues $\epsilon_{\nu}(\vec{k})$, where $\nu$ is the band index, whose loci in $\epsilon-\vec{k}$ space trace the conventional electronic structure. For ordered systems this leads to

$$
S^{2}(\vec{Q}) \sim \sum_{\nu, \nu^{\prime}} \sum_{\vec{k}} \frac{f\left(\epsilon_{\nu}(\vec{k})\right)-f\left(\epsilon_{\nu^{\prime}}(\vec{k}+\vec{Q})\right)}{\epsilon_{\nu}(\vec{k})-\epsilon_{\nu^{\prime}}(\vec{k}+\vec{Q})},
$$

and has a susceptibility form[4][5]. The principal contributions arise when $\epsilon_{\nu}(\vec{k})$ are close to the Fermi energy (Fermi energy is where the highest electronic 


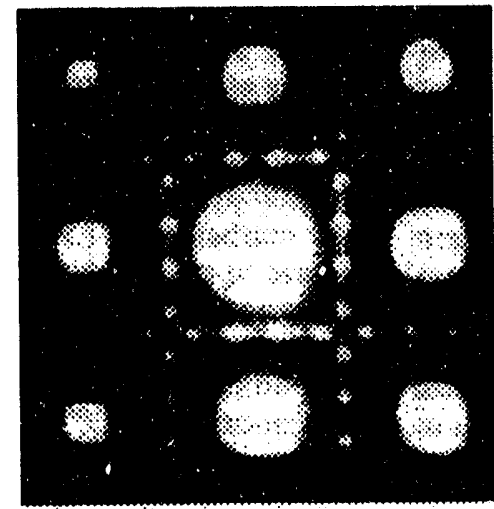

Figure 4: Electron diffuse scattering intensities for 19 at.\% $\mathrm{Mg}$.

states are occupied). In addition, $S^{2}(\vec{Q})$ will be particularly large for spanning vectors $\vec{Q}$ that connect two well-defined parallel sheets of Fermi surface, given by $A^{B}\left(\vec{k}, \epsilon_{f}\right)$ and comes from the fact that $\epsilon_{\nu}(\vec{k})-\epsilon_{\nu^{\prime}}(\vec{k}+\vec{Q})=0$ in the above equation. A large value for $S^{2}(\vec{Q})$ at specific $\vec{Q}$ suggests the system is susceptible to small changes by an external field and is a signature of an incipient instability. The instability is driven by the creation of a large number of electron-hole pairs (via the external field) with wave vector $\vec{Q}$ and approximately zero energy. The electrons are excited from just below one of the Fermi surface sheets to the previously empty states just above the other sheet. The Fermi surface mechanisms give rise to several types of instabilities such as charge density waves[28], spin density waves[2] and, specifically for this investigation, concentration waves[14].

\section{Results and Discussion}

Displayed in figure 4 is the electron diffuse scattering experiments for the $\mathrm{Ag}-\mathrm{Mg}$ alloy at 19 at. \% as measured by Ohshima and Watanabe [21]. The large spot at the center is the origin $(000)$, the uppermost right spot is at the $(220)$ position $(\langle 110\rangle$ direction) and the large spot to the right of the origin is the $(200)$ position ( $(100\rangle$-direction). Note, along the $\langle 110\rangle$-direction the appearence of a four-fold satellite peak and a two-fold satellite peak along the $\langle 100\rangle$ direction. Recall, for the homogeneously random alloy the background of the diffuse scattering intensity is unmodulated (the occupancy of sites is uncorrelated). The appearence of these satellite peaks means that the occupancy of the sites are correlated. This is a signature of short-range ordering process that is found at high-temperatures [21].

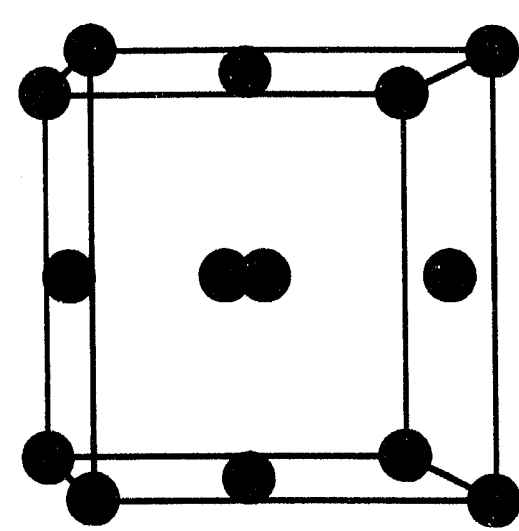

Fiqure 5: FCC Crystal Structure

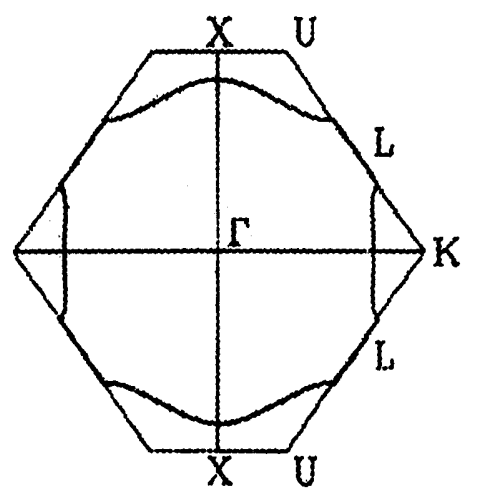

Figure 6: Fermi surface for 20 at.\% Mg.

We have determined the electronic structure for several concentrations ranging from 10-30 at.\% of the correspondingly randomly disordered face-centered cubic (fcc) crystal structure (see figure-5).

The minimization of the total energy with respect to the lattice constant was performed for all compositions. The self-consistent potentials for the fcc disordered solid solution were used to calculate the Fermi surface for each concentration by calculating $A^{B}(\vec{k}, \epsilon)$ at the Fermi energy. The Fermi-surface topology was similar for all compositions. The Fermi surfaces of the alloys were found to be well-defined with a broadening due to the disorder to be less than $1 \%$ of the Brillouin zone dimension. The sharpness of the Fermi surfaces means that it can be accurately represented in $\vec{k}$ space by the loci of the maxima in $A^{B}(\vec{k}, \epsilon)$. In figure 6 we show a particular plane of the Fermi surface calculated for 20 at. $\% \mathrm{Mg}$.

The Fermi surface of $\mathrm{Ag}-\mathrm{Mg}$ at 20 at. $\% \mathrm{Mg}$ is quite flat between the L-points, along the $\langle 110\rangle$ directions. This feauture is common to to all compositions investigated, differing only in the length of the spanning vector $\vec{Q}$. The length of $\vec{Q}$ increases with increasing $\mathrm{Mg}$ concentration. As the concentration of $\mathrm{Mg}$ is increased the electron-per-atom ratio increases with a corresponding increase in the size of the Fermi surface, leading to an increase in the length of $\vec{Q}$. Because the 


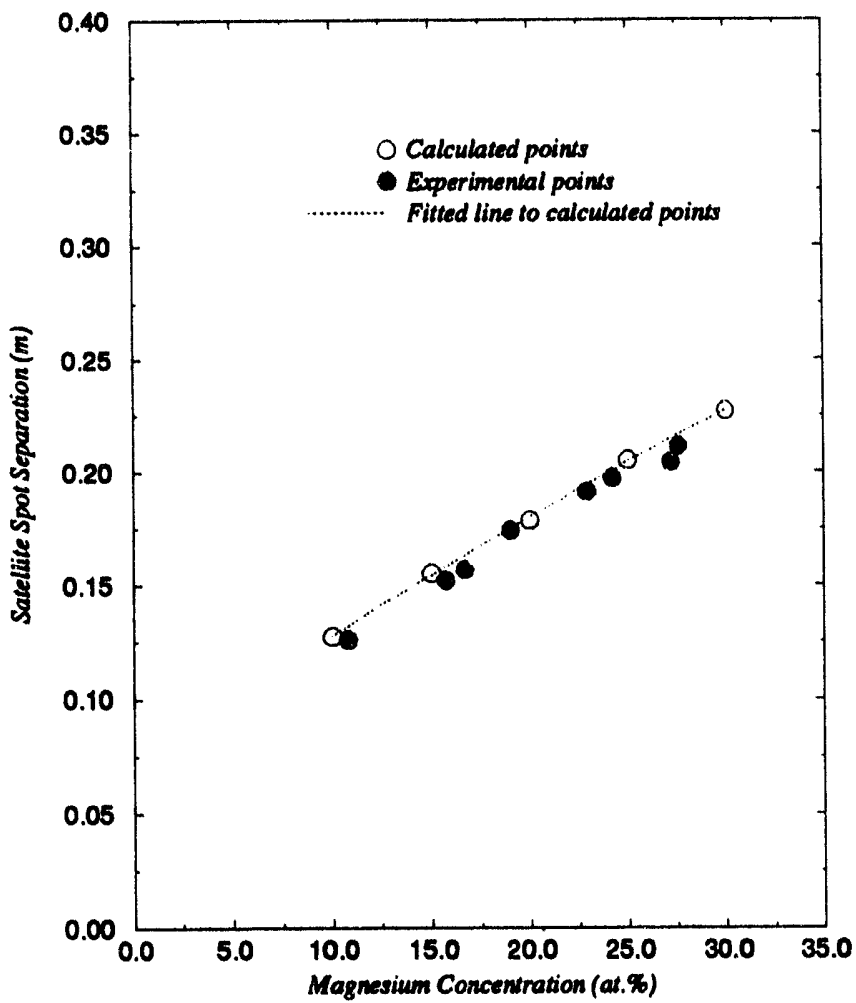

Figure 7: Experimental and calculated variation of electron diffuse scattering satellite spot separation for disordered Ag-Mg alloys with composition.

length of $\vec{Q}$ is composition dependent, means that the spot separation of the satellite peaks also depends on the $\mathrm{Mg}$ concentration. Ohshima and Watanabe. [21] measured the distance $m$ between spots for a various concentrations. In figure 7 we compare their results with our calculated values, The agreement between theory and experiment is excellent.

The flatness of the Fermi surface should lead to a peak in $S^{2}(\vec{q})$ at $\vec{q}=\vec{Q}$. The positions of $I(\vec{Q})$ can be obtained by calculating $S^{2}(\vec{q})$. We have calculated the diffuse scattering intensity of $\mathrm{Ag}_{75} \mathrm{Mg}_{25}$ using the $S^{2}(\vec{q})$ formalism of Staunton et al. [27,11] as shown in figure 8. Indeed, the results are from Fermi-surfice nesting and give excellent agreement with experimeni. Furthermore, the calculated instability temperature (i.e. spinodal) is $670 \mathrm{~K}$, which may be compared to the actual order-disorder temperature of $665 \mathrm{~K}$.

This provides strong evidence that the short-range order in $\alpha$-phase $\mathrm{Ag}-\mathrm{Mg}$ is Fermi surface driven and demonstrates the accuracy of the KKR-CPA shortrange order calculations in treating the phenomenon of incommensurate concentration waves.

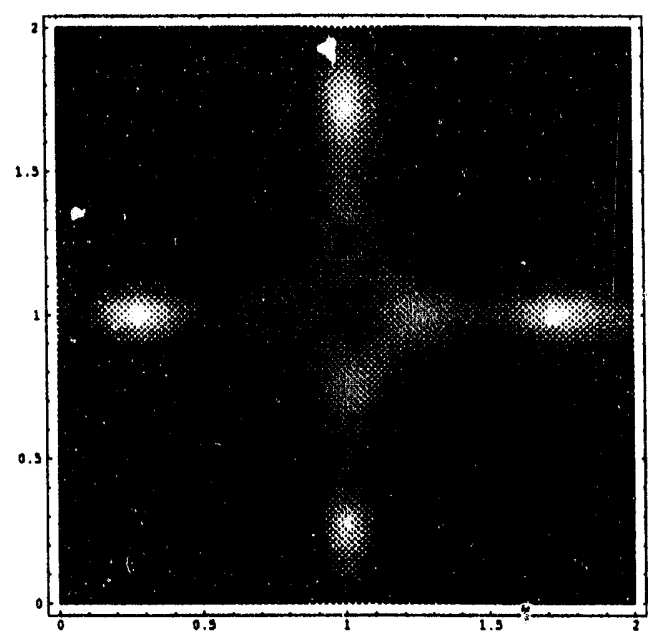

Figure 8: $S^{2}(\vec{q})$ calculated electron diffuse scattering intensity for $\mathrm{Ag}_{75} \mathrm{Mg}_{25}$

\section{Conclusion}

We have calculated the Fermi surfaces of $\alpha$-phase Ag-Mg alloys in the composition range of $10-30$ at.\% $\mathrm{Mg}$ using the LDA-SCF-KKR-CPA method. The Fermi surfaces were discovered to be well defined with flattened regions normal to the $\langle 110\rangle$ directions. These regions produce a maximum effect in the direct correlation function $S^{2}(\vec{q})$, at $\vec{q}=\vec{Q}$, where $\vec{Q}$ is the spanning vector connecting two flat parallel sheets of Fermi surface. This behavior accounts for the satellite peaks observed in electron diffuse scattering experiments. In addition, the variation of the Fermi-surface spanning vector with $\mathrm{Mg}$ concentration accounts for the spot separation (m) with $\mathrm{Mg}$ concentration. The shortrange order (or concentration waves) in $\alpha$-phase Ag$\mathrm{Mg}$ alloys is driven by the Fermi-surface topology. Recently Jordan et al. [12] have argued that this Fermisurface topolgy is responsible for the modulation of the $L 1_{2}$ ordered $\alpha$ phase [13], i.e. the long-period superlattice modulation in the ordered alloys near the $\mathrm{Ag}_{3} \mathrm{Mg}$ composition and the concentration variation of the period. The calculated short-range order and its agreement with experiment, as well as the concentration dependence of the diffuse spots, lends strong support to this suggestion. This illustrates the important role that first-principles, electronic-structure calculations play in determining the properties of alloys. 


\section{Acknowledgements}

Work supported ty the Applied Mathematical Sciences Research Program (WAS and GMS), Office of Energy Research, US Department of Energy subcontract DEAC05-840R21400 with Martin-Marietta Energy Systems, Inc., (RGJ and LQ), from the National Science Foundation, contract DMR-9120120, from the Southeastern Universities Research Association (RGJ) as part of the SURA/ORNL material program. For DDJ and FJP, work supported by US Department of Energy, Basic Energy Sciences, Division of Materials Science under a New Initiative at Sandia (\#DE-AC0494AL85000). JBS support is from the S.E.R.C. in the U.K.

\section{References}

[1] J. M. Cowley, Diffraction Physics, NorthHolland, New York, New York, 1981.

[2] E. Fawcett, Rev. Mod. Phys., 60 (1988), p. 209.

[3] D. D. Fontaine, in Solid-State Physics, H. Ehrenreich, F. Seitz, and D. Turnbull, eds., vol. 34, Academic Press, New York, NY 10003, 1979 , p. 73.

[4] B. L. Gyorffy and et. AL., in Alloy Phase Stability, G. M. Stocks and A. Gonis, eds., vol. 163, Dordrecht, Netherlands, 1989, NATO ASI Series E: Applied Sciences, Kluwer Academic Publishers, p. 421.

[5] —, in Alloy Phase Stability and Design, G. M. Stocks, D. P. Pope, and A. F. Giamei, eds., vol. 186, Pittsburgh, PA 15237, 1991, Materials Research Society, p. 3.

[6] B. L. Gyorffy and G. M. Stocks, Phys. Rev. Lett., 50 (1983), p. 374.

[7] P. Hohendera and W. Kohn, Phys. Rev., 136 (1964), p. 864.

[8] D. D. Johnson, D. M. Nicholson, F. J. PinSKI, B. L. GyGrFfy, AND G. M. Stocks, Phys. Rev. Lett., 56 (1986), p. 2088.

[9] — Phys. Rev. B, 41 (1990), p. 9701.

[10] D. D. Johnson And F. Pinski, Phys. Rev. B, 48 (1993), p. 11553.

[11] D. D. Johnson, J. B. Staunton, and F. PinSKI, submitted Phys. Rev. B, (1994).
[12] R. G. Jordan and Et. Al., Phys. Rev. B, 47 (1993), p. 16521.

[13] —, in press Acta. Met., (1994).

[14] A. G. Khachaturyan, Theory of Structural Phase Transformations in Solids, John Wiley and Sons, New York, 1983.

[15] - Theory of Structural Transformations in Solids, Wiley, New York, 1983.

[16] W. Kohn and N. Rostoker, Phys. Rev., 94 (1954), p. 1111.

[17] W. Kohn and L. J. Sham, Phys. Rev., 140 (1965), p. 1133.

[18] J. Korringa, Physica, 13 (1947), p. 392.

[19] E. M. Lifshicz and L. P. Pitaevskil, Statistical Physics, Part 1, Pergamon Press, Elmsford, New York 10523, Third edition, 1980.

[20] R. Mills, L. J. Gray, and T. Kaplan, Phys. Rev. B, 27 (1983), p. 3252.

[21] K. Ohshima and D. Watanabe, Acta Crystallogr, A33 (1977), p. 784.

[22] A. Pindor, B. L. Gyorffy, And W. M. Temmerman, Joural of Physics F: Metal Physics, 13 (1983), p. 1627.

[23] F. J. Pinski, D. M. Nicholson, G. M. Stocks, W. Butler, D. D. JohNSON, AND B. L. GYoRfFY, in Third International Conference on Supercomputing, L. Kartashev and S. Kartashev, eds., vol. 1, 1988, p. 321.

[24] L. Schwartz and E. Siggia, Phys. Rev. B, 5 (1972), p. 383.

[25] W. A. Shelton, (1989). PhD Thesis: University of Cincinnati (unpublished).

[26] P. Soven, Phys. Rev., 156 (1967), p. 809.

[27] J. B. Staunton, D. D. Johnson, And F. PinSKI, submitted Phys. Rev. B, (1994).

[28] J. A. Wilson, F. J. D. Salvo, and F. MahaJAN, Adv. Phys., 24 (1975), p. 117. 

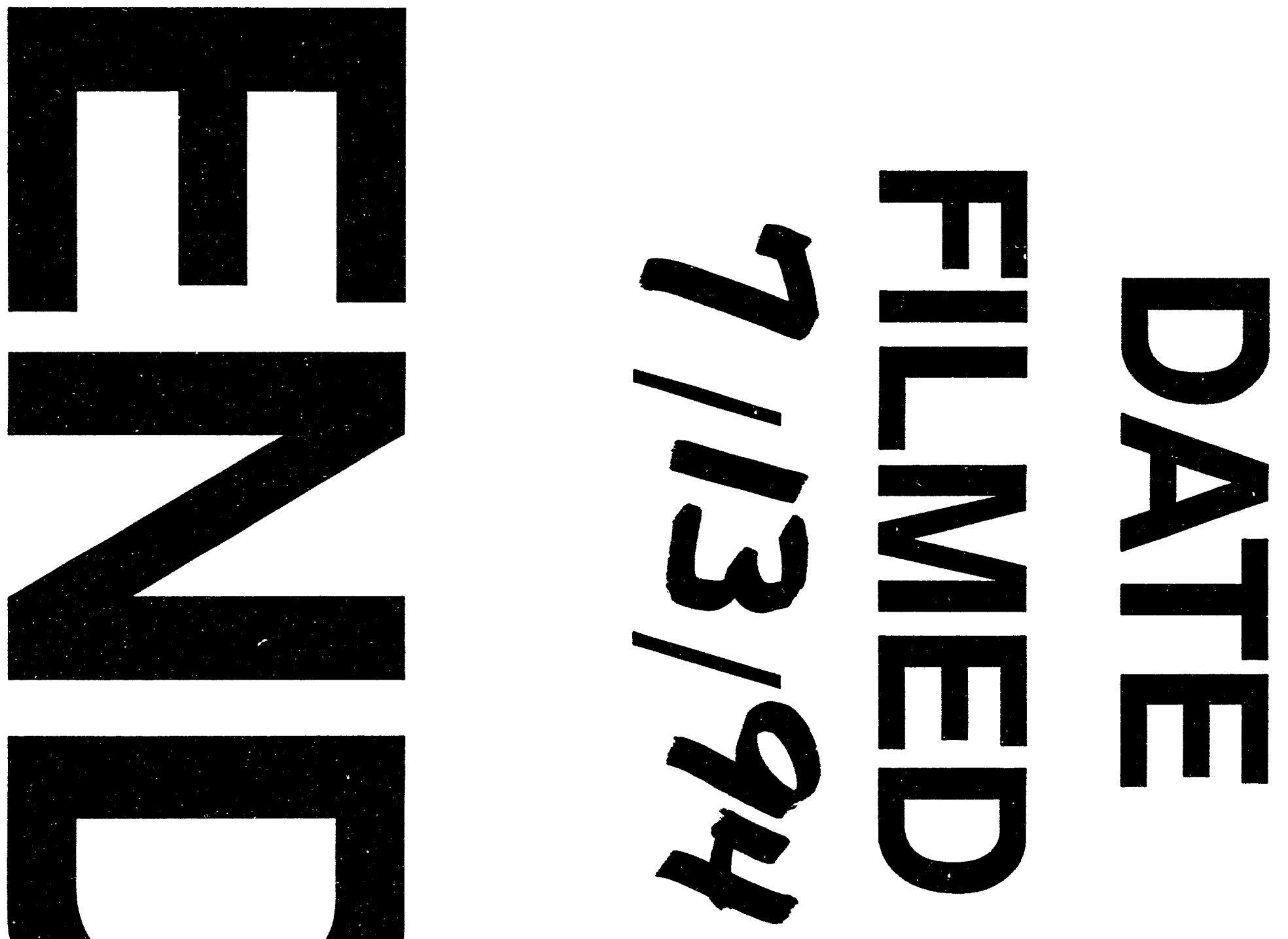

$-6$

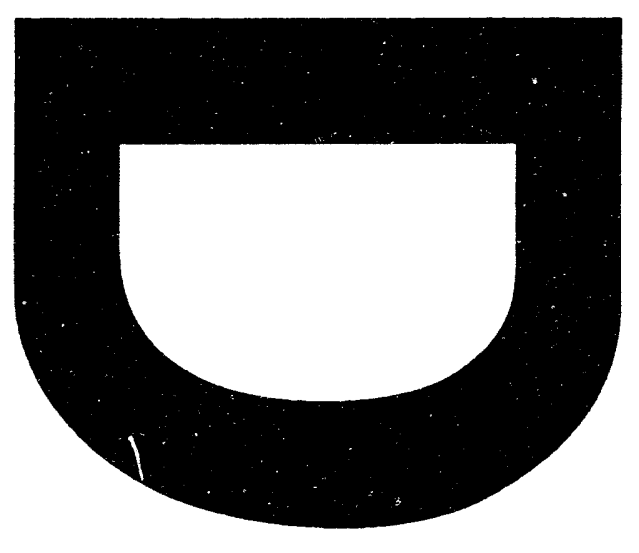


